\title{
Reconstruction of Head and Neck Defects with Lower Trapezius Myocutaneus Flap
}

\author{
Somesh Mozumder, ${ }^{1}$ Shirish Dubey, ${ }^{1}$ Kinshuk Chatterjee, ${ }^{1}$ Priyadarshan Kumar, ${ }^{1}$ Ankit Khandelwal, ${ }^{1}$ Aniruddha Dam, ${ }^{1}$ Anup \\ Kumar Bhowmick
}

Introduction:

ABSTRACT

High lateral and posteriorly based defects are challenging to reconstruct as mobilization of conventional pedicled flaps is difficult. This study was done to evaluate the usefulness of lower trapezius myocutaneous flap (LTMC) in selected cases as a reconstructive alternative to other pedicled flaps which have positional and technical disadvantages and/or in cases where free flap is not possible.

\section{Materials and Methods}

Ten cases of locally advanced (T3 and T4) high and laterally placed head and neck carcinoma (8 cases of SCC involving posterior scalp, ear lobule, skin anterior to tragus and 2 cases of locally advanced salivary gland malignancies involving parotid glands) irrespective of sex had been selected. Due to non-availability of plastic surgeon in the institute none of the patient could be subjected to free flap reconstruction. All the patients received post operative adjuvant radiotherapy and were followed up on a monthly basis for six months at least.

\section{$\underline{\text { Results }}$}

Out of total 10 cases 5 underwent wound infection and dehiscence at recipient site. In 3 cases there were donor site seroma. In 2 cases healing was uneventful. However in all cases the flap was healthy and flap survival was 100\%. There was no flap contracture in long term follow up.

\section{Conclusion}

The LTMC flap is ideal for mentioned defects because of its anatomical location, abundant blood flow, minor donor-site morbidity, and long pedicle. The LTMC flap, though less commonly used, is a precious option in situations where free flaps and other pedicled flaps are not feasible.

Keywords:

Trapezius; Myocutaneus Flap

$\mathrm{H}$ igh lateral and posteriorly based head and neck defects are challenging to reconstruct as conventional pedicled flaps i.e. Deltopectoral fasciocutaneous flap or Pectoralis Major myocutaneus flaps are very difficult to mobilise to these locations. In these scenarios free flaps are currently considered the preferred choice for reconstruction. However, medical co-morbidities, unavailability of plastic surgeons

1 - Department of ENT, Chittaranjan National Cancer

Institute, Kolkata

\section{Corresponding author:}

Dr Somesh Mozumder

email: drsomeshmozumder@gmail.com sometimes, treatment with prior chemoradiotherapy, (often associated with a poor general and local condition that may impair the healing process and reduce tolerance of surgical stress) and failure of previously done free flap (i.e. anterolateral thigh flap etc) make many head and neck cancer patients ineligible for free flap reconstruction.

Pedicled flap surgery should be considered in this context. An ideal flap would meet the following requirements: one-step resection and reconstruction, ease and feasibility of use, short surgical duration, low complication rate, and satisfactory functional results. The authors recommend performing reconstruction using lower trapezius musculocutaneous (LTMC) in such situations. The LTMC flap enables successful 
reconstruction without severe complications, demonstrating the advantages and usefulness of such surgery. ${ }^{1-3}$

\section{Materials and Methods}

The study was conducted from December 2015 to November 2016 to evaluate the usefulness of lower trapezius myocutaneous flap in selected cases of high and laterally placed head and neck defects as a reconstructive alternative to other pedicled flaps which have positional and technical disadvantages and/or in cases where free flap is not possible.

10 cases of locally advanced (T3 and T4) high and laterally placed head and neck carcinoma (8 cases of SCC involving posterior scalp, ear lobule, skin anterior to tragus and 2 cases of locally advanced salivary gland malignancies involving parotid glands) irrespective of sex had been selected. (Fig.1)

Patients below 18 years and above 70 years were excluded from study as extremes of age are not very good candidates for prolonged and extensive resections and reconstructions. Due to non-availability of plastic

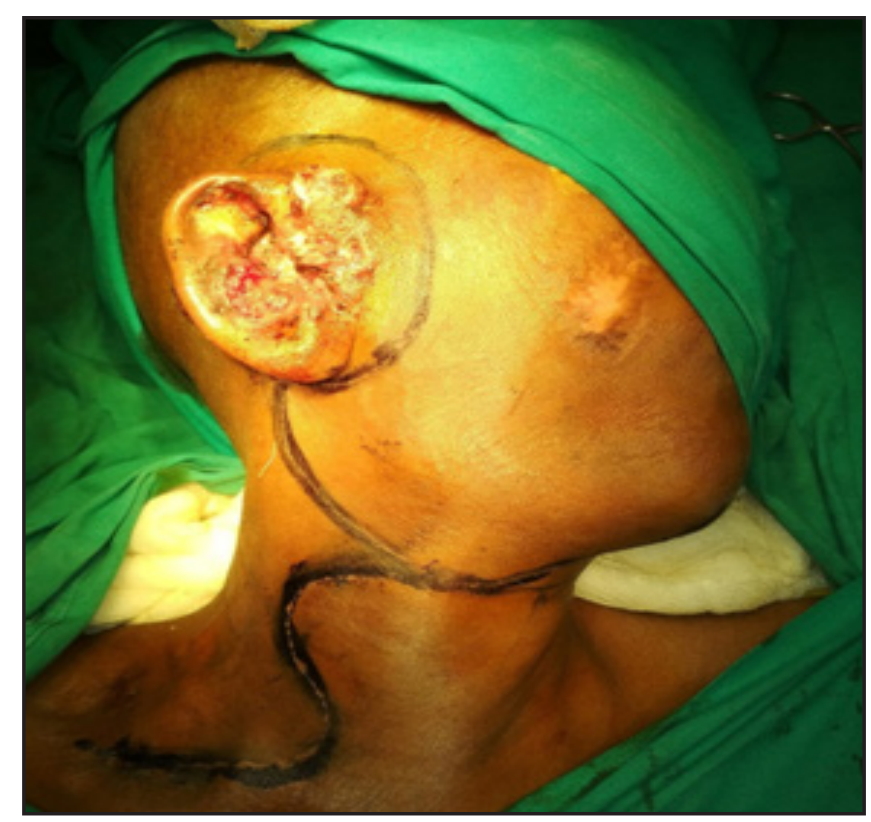

Fig.1. A case of Squamous cell ca involving right ear lobule with extension to pre-aural region and adjacent parotid gland surgeon in the institute none of the patient could be subjected to free flap reconstruction. All patients were otherwise physically fit (E-Cog: 0 or 1), devoid of any medical co morbidities and were fit for general anaesthesia. Proper staging and resectabilty of each case was assessed radiologically (with contrast enhanced CT Scan or MRI) before operation.

All patients underwent a single stage reconstruction in same sitting, using lower trapezius myocutaneous flap after primary resection and neck dissection. Average time for resection and reconstruction was $6 \mathrm{hrs}$ out of which around 2 hours were taken for flap harvesting and reconstruction.

Harmonic scalpel was used for flap harvesting which significantly reduced time and minimised blood loss.

All the patients received post operative adjuvant radiotherapy and were followed up on a monthly basis for 6 months at least.

Surgical anatomy and gross steps of reconstruction using lower trapezius myocutaneous flap:

After primary resection and neck dissection (Fig. 2), patient is put to prone position with arm adducted and palm looking anteriorly to make scapular medial border

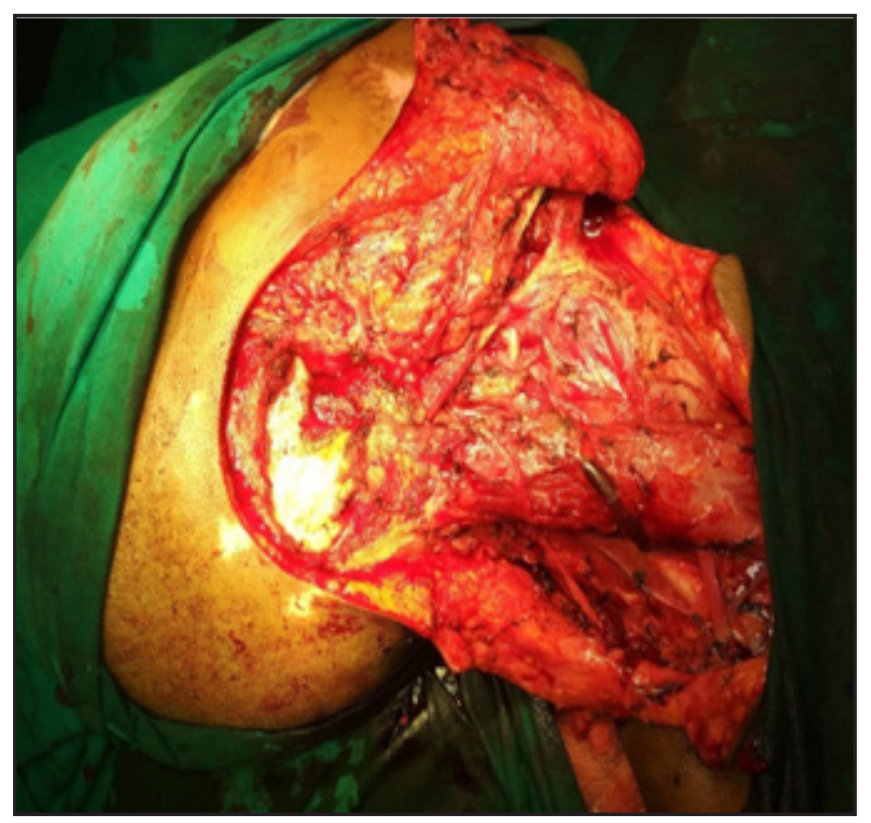

Fig. 2. After wide local excision of mass with mastoidectomy 


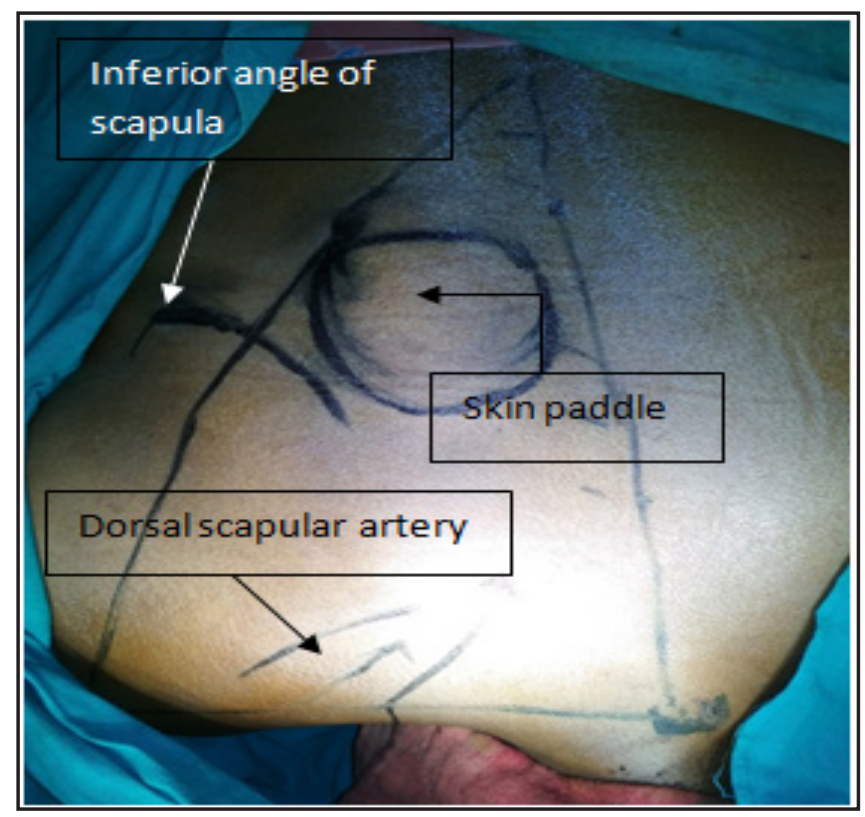

Fig. 3. Marking the LTMC flap

prominent. The fan shaped trapezius flap is outlined by drawing a triangle comprised of a horizontal line drawn from acromion process of scapula to $\mathrm{T} 1$ vertebral spine superiorly; another line drawn from acromion process passing inferomedially, medial to inferior angle

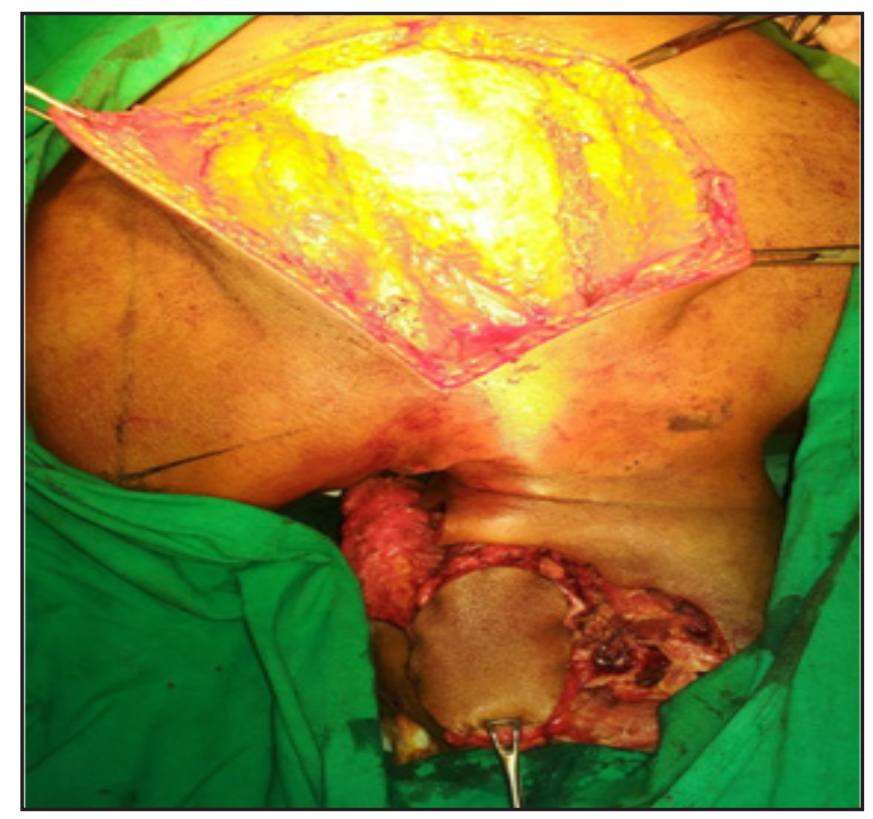

Fig. 5. Delivery of flap to recipient area

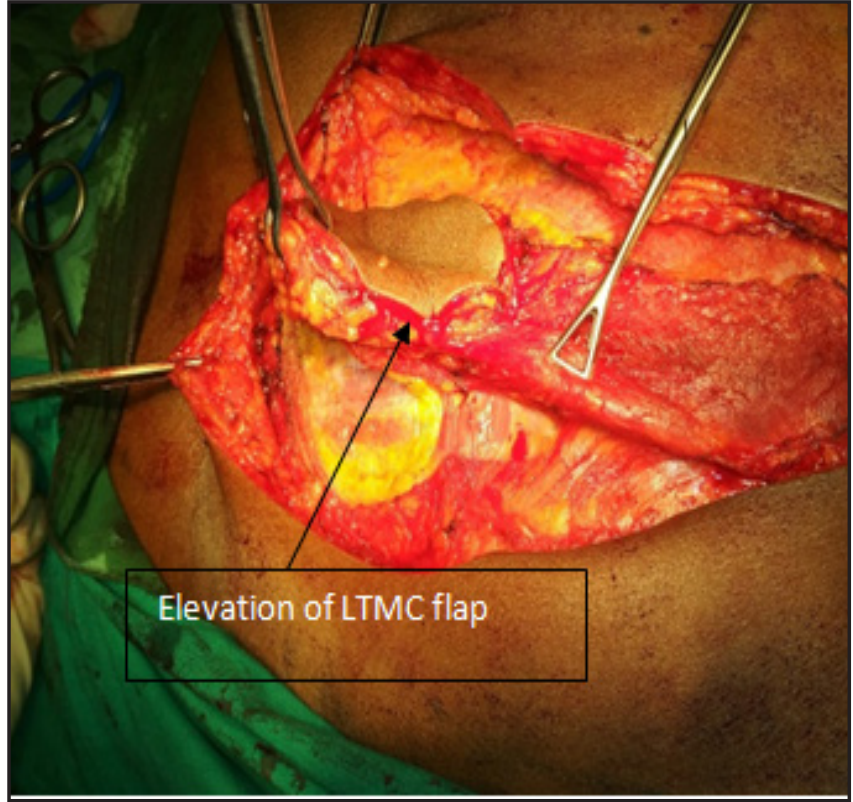

Fig. 4. Elevation of LTMC flap

of scapula up to spine of T12 vertebral and medial boundary by a line passing along the spine which is intersected by the above two.

The skin paddle is marked according to the size of defect (not less than 4 finger breadth, to avoid vascular

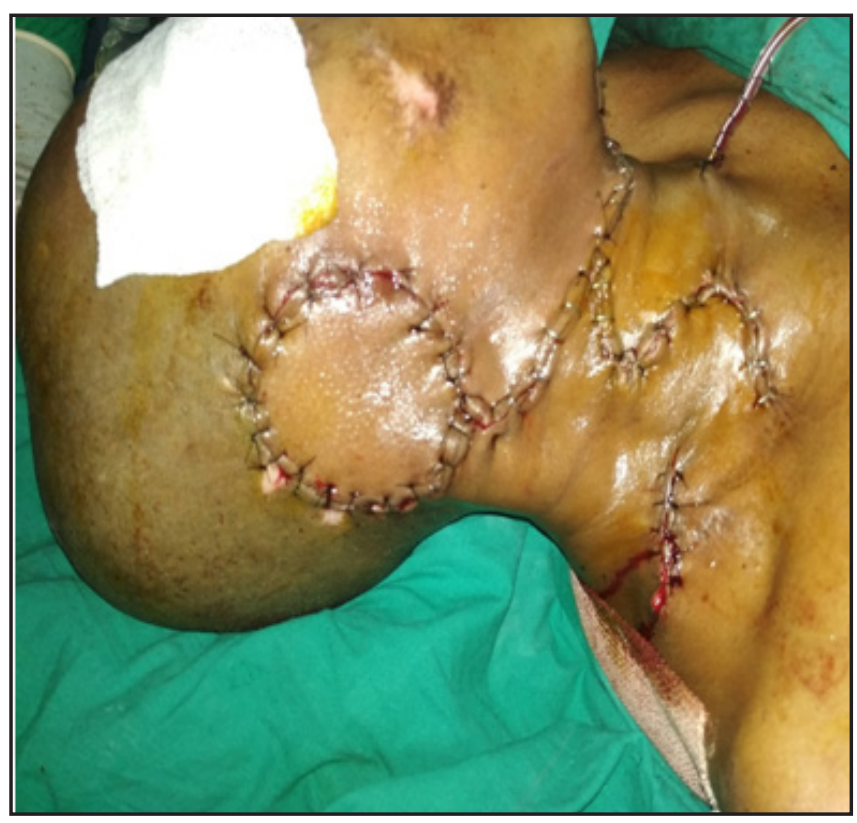

Fig. 6. After final reconstruction 


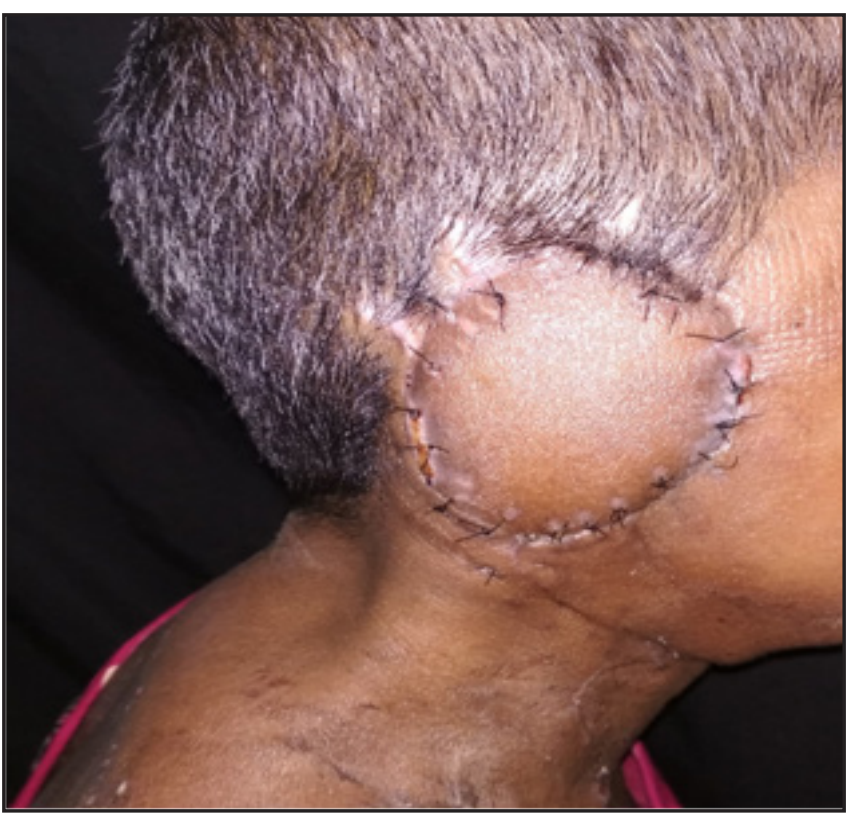

Fig. 7. 10 days after operation

compromise of skin paddle, which is supplied by perforators through muscle) and is drawn between spine and medial border of scapula and can be extended up to $5 \mathrm{~cm}$ below inferior angle of scapula.

The blood supply of the trapezius muscle is one of the most confusing of any of the regional flaps. The main blood supply is classically supposed to be via the transverse cervical artery (TCA) and vein. TCA after origin from the thyrocervical trunk divides into a superficial branch and a deep branch. The superficial branch with its divisions together with the occipital artery supplies the superior portions of the muscle.

The deep branch in most cases has an independent origin and is known as dorsal scapular artery (DSA). DSA may originate from TCA or thyrocervical trunk or the first or second part of the subclavian. Thus TCA and DSA both supply the muscle and in lower part either may be used as the pedicle. This pedicle enters the under surface of the muscle approximately at mid-point of upper horizontal arm of triangle medial to scapula is also marked. (Fig.3)

Vertical incisions above and below skin paddle is given. Skin, subcutaneous tissue is cut exposing the muscle. Skin flaps are raised on either side, exposing spine medially and medial border of scapula laterally to identify the extent of muscle before elevation. The lower trapezius myo-cutaneus flap is elevated in superior direction carefully and gently keeping an eye to the under surface to avoid pedicle injury. A small twig arising from dorsal scapular artery and going deep between Rhomboideus Major and Minor is ligated and cut to facilitate further elevation of flap superiorly and above spine of scapula. (Fig.4) The flap is passed into neck underneath the skin. (Fig.5)

Primary closer of donor site defect is done after achieving hemostasis and putting suction drain. Patient is again put to supine position. Operative site defect is closed by the flap. Neck is closed after achieving hemostasis and putting suction drain. (Figs.6 \& 7)

\section{Results}

Out of 10 cases there was no incidence of total flap failure; though in 5 cases (50\%) wound infection and partial dehiscence at recipient site was noted which was controlled by judicious use of antibiotics, regular sterile dressing and secondary suturing after controlling local infection. ${ }^{4}$

Donor site seroma occurred in another 3 cases (40\%) which was controlled conservatively using compressing bandage and suction drain. ${ }^{4}$

For two flaps the entire course of healing during post operative period was uneventful and uncomplicated.

After adjuvant radiotherapy, during the course of long term follow up (for at least 6 months), flaps remained healthy with no contracture, and acceptable colour and texture.

\section{Discussion}

The trapezius muscle is superficial, flat, triangular, and large in size; it covers the posterior cervical and interscapular thoracic areas. Along with the pectoralis and rotator cuff muscles, the trapezius suspends the pectoral girdle from its muscular origin located along the external occipital protuberance, the medial third of the superior nuchal line, the ligament nuchae, and the spinous processes of the seventh cervical and twelfth thoracic vertebrae. ${ }^{5}$ According to the classification of Mathes and Nahai, ${ }^{6}$ the trapezius muscle has a type II 
vascular pattern with dominant and additional minor vascular pedicles. The transverse cervical artery with its branches is the major feeding artery. The additional minor vascular pedicles are the occipital artery and posterior intercostal artery.

Functionally and aesthetically, the muscle may be considered to have an upper and a lower portion. The upper portion of the muscle inserts along the lateral third of the clavicle. It receives the majority of its blood supply from the transverse cervical artery. It also contains the spinal accessory nerve, which courses along its deep surface and provides motor innervations to the entire muscle. The lower portion of the muscle inserts along the lateral spine of the scapula; it is a dispensable subunit. The superficial and deep branches of the transverse cervical artery supply blood to this portion of the muscle. ${ }^{7-12}$ The distinction between the upper and lower parts of the trapezius allows the lower portion of the muscle to be used for wound coverage while preserving normal shoulder function. ${ }^{13}$

The authors used a LTMC flap with the dorsal scapular artery and the deep branch of the transverse cervical artery, as a pedicle for reconstruction. This flap can be elongated up to $5 \mathrm{~cm}$ (measured from the tip of the scapula) with minimal damage to the nerve and with preservation of the muscle fibers from the proximal trapezius muscle. Moreover, if the pedicle is damaged during surgery, it can be replaced with the superficial cervical artery. ${ }^{14,15}$ The LTMC flap is thin and pliable, and also has a very long constant pedicle and minor donor-site morbidity.

Reconstruction of a head and neck defect with a local flap in combination with planned radiation therapy may delay wound healing, and a free flap might cause severe morbidity in patients with a poor general condition or those with a tumor with a poor prognosis. ${ }^{1,16}$ Thus, if a one-step reconstruction is planned for such patients, regional flap surgery is preferable. Other regional flaps include the pectoralis major musculocutaneous flap and the supraclavicular artery island flap, deltopectoral fasciocutaneous flap. The pectoralis major musculocutaneous flap provides thick muscle volume so it can protect the important neck organs, but it also creates an abnormal bulge at the root of the neck that causes neck stiffness. ${ }^{2,17}$ The supraclavicular artery island flap is near the recipient site; thus, flap color and texture are very similar to that of the recipient site. However, because it has a short pedicle, it can cover only the lower border of the face and neck. ${ }^{3,18}$ Deltopectoral fasciocutaneous flap and pectoralis major musculocutaneous flaps are very difficult to move to high lateral and posteriorly placed head and neck defects.

\section{Conclusion}

LTMC flap has a wide arc of rotation because of long constant pedicle, low donor-site morbidity, richly vascularized, and ensure serviceable skin coverage of areas as far cephalic as the vertex of the skull without evidence of tension or traction. Our technique clearly show that this less commonly used flap is particularly useful in patients with high lateral and/or posteriorly placed defects for whom neither free flap surgery is possible because of co-morbidities, unavailability of technical expertise, and previous radiation injury and previous free flap failure etc. nor deltopectoral fasciocutaneous flap, pectoralis major musculocutaneous flap or supraclavicular artery island flaps can be done because of their unfavorable anatomical location to move to high lateral and posteriorly placed head and neck defects.

This is a hardy and reliable flap in our experience, with minimum early postoperative complications and excellent long term outcome which is quite acceptable when compared to the same of other flaps. More over in comparison to free flaps like anterolateral thigh flap; it has less chances of post operative flap failure as the vascular pedicle remains attached to donor site where as in free flaps the supplying vessels are cut and re-ligated with new vessels near recipient area, thus bearing the risk of thrombosis and bleeding from anastomosis site. Post operative care and management is relatively easier than free flaps. Operative time is also much less than free flaps there by chances of complications like deep vein thrombosis( DVT) or pulmonary thromboembolisation( PTE ) is also minimum.

The only disadvantage is that the patient's position has to be changed twice during the operation for flap harvesting and patient has to bear another suction drain at his/her back for a few days which is pretty 
uncomfortable.

Therefore to conclude with; though less commonly used, lower trapezius musculocutaneous (LTMC) can be a precious option in the arsenal of head and neck reconstruction in difficult situations.

\section{References}

1. Choi EC, Kim CH, Kim SH, et al. The role of pectoralis major myocutaneous flap in the era of free flap. Korean J Head Neck Oncol. 2001; 17:190-3

2. Ariyan S. The pectoralis major myocutaneous flap. A versatile flap for reconstruction in the head and neck. Plast Reconstr Surg. 1979; 63:73-81

3. Pallua N, Magnus Noah E. The tunneled supraclavicular island flap: an optimized technique for head and neck reconstruction. Plast Reconstr Surg. 2000; 105:842-51

4. Ou KL, Dai YH, Wang HJ, et al. The lower trapezius musculocutaneous flap for head and neck reconstruction: two decades of clinical experience. Ann Plast Surg. 2013;71 Suppl 1:S48-54

5. Netterville JL, Wood DE. The lower trapezius flap. Vascular anatomy and surgical technique. Arch Otolaryngol Head Neck Surg. 1991; 117:73-6

6. Mathes SJ, Nahai F. Classification of the vascular anatomy of muscles: experimental and clinical correlation. Plast Reconstr Surg. 1981; 67:177-87

7. Mathes SJ, Nahai F. Clinical atlas of muscle and musculocutaneous flap. St. Louis: Mosby; 1979

8. McCraw JB, Dibbell DG. Experimental definition of independent myocutaneous vascular territories. Plast Reconstr Surg. 1977; 60:212-20

9. McCraw JB, Dibbell DG, Carraway JH. Clinical definition of independent myocutaneous vascular territories. Plast Reconstr Surg. 1977; 60:341-52

10. Urken ML, Naidu RK, Lawson W, et al. The lower trapezius island musculocutaneous flap revisited. Report of 45 cases and a unifying concept of the vascular supply. Arch Otolaryngol Head Neck Surg. 1991; 117:502-11

11. Baek SM, Biller HF, Krespi YP, et al. The lower trapezius island myocutaneous flap. Ann Plast Surg. 1980; 5:108-14

12. Haas F, Weiglein A, Schwarzl F, et al. The lower trapezius musculocutaneous flap from pedicled to free flap: anatomical basis and clinical applications based on the dorsal scapular artery. Plast Reconstr Surg. 2004; 113:1580-90

13. Lynch JR, Hansen JE, Chaffoo R, et al. The lower trapezius musculocutaneous flap revisited: versatile coverage for complicated wounds to the posterior cervical and occipital regions based on the deep branch of the transverse cervical artery. Plast Reconstr Surg. 2002; 109:444-50

14. Angrigiani C, Grilli D, Karanas YL, et al. The dorsal scapular island flap: an alternative for head, neck, and chest reconstruction. Plast Reconstr Surg. 2003; 11:67-78

15. Tan KC, Tan BK. Extended lower trapezius island myocutaneous flap: a fasciomyocutaneous flap based on the dorsal scapular artery. Plast Reconstr Surg. 2000; 105:1758-63

16. Ugurlu K, Ozcelik D, Huthut I, et al. Extended vertical trapezius myocutaneous flap in head and neck reconstruction as a salvage procedure. Plast Reconstr Surg. 2004; 114:339-50

17. Ariyan S. Further experiences with the pectoralis major myocutaneous flap for the immediate repair of defects from excisions of head and neck cancers. Plast Reconstr Surg. 1979; 64:605-12

18. Chiu ES, Liu PH, Friedlander PL. Supraclavicular artery island flap for head and neck oncologic reconstruction: indications, complications, and outcomes. Plast Reconstr Surg. 2009; 124:115-23. 